UCRL-JC-127394

Preprint

\title{
A Comparison of Neutral Gas Models for Divertor Plasmas
}

\author{
M.E. Rensink, L. Lodestro, G.D. Porter, T.D. Rognlien, and D.P. Coster
}

This paper was prepared for submittal to

6th International Workshop on Plasma Edge Theory In Fusion Devices

Oxford, England

September 15-17, 1997

September 10, 1997

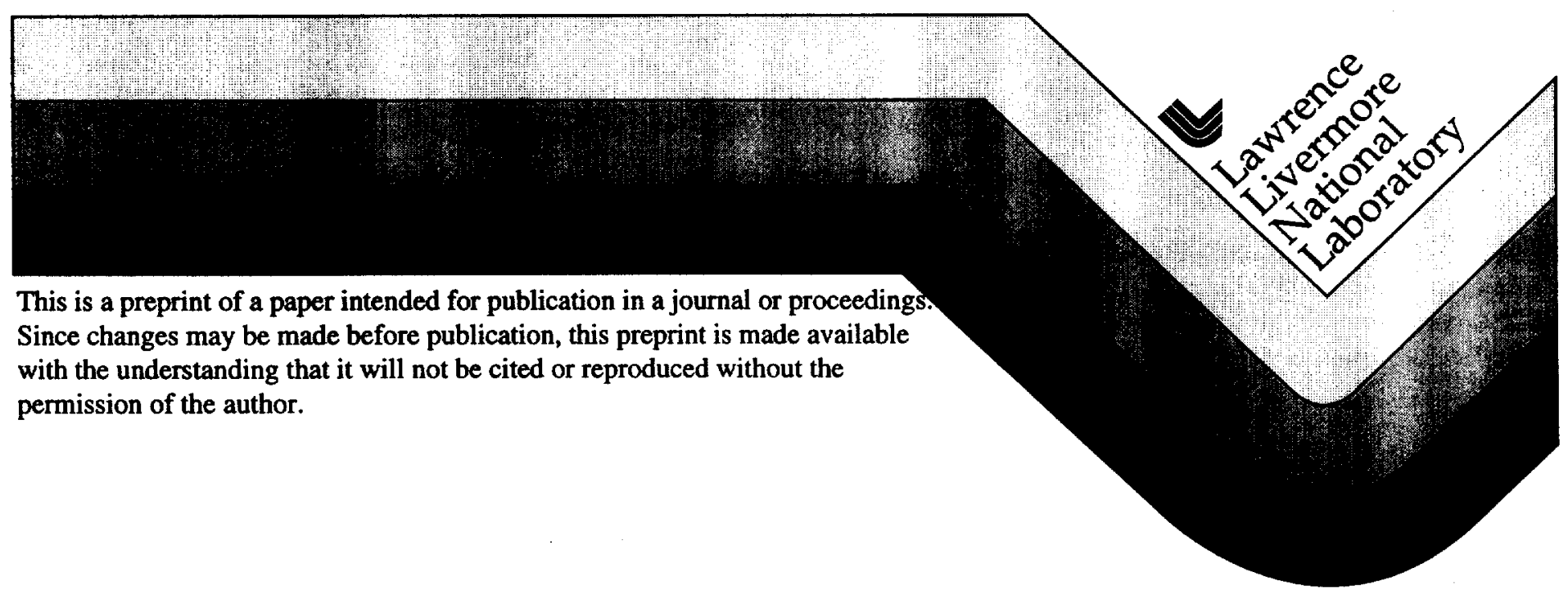




\section{DISCLAMMER}

This document was prepared as an account of work sponsored by an agency of the United States Government. Neither the United States Government nor the University of California nor any of their employees, makes any warranty, express or implied, or assumes any legal liability or responsibility for the accuracy, completeness, or usefulness of any information, apparatus, product, or process disclosed, or represents that its use would not infringe privately owned rights. Reference herein to any specific commercial product, process, or service by trade name, trademark, manufacturer, or otherwise, does not necessarily constitute or imply its endorsement, recommendation, or favoring by the United States Government or the University of California. The views and opinions of authors expressed herein do not necessarily state or reflect those of the United States Government or the University of California, and shall not be used for advertising or product endorsement purposes. 


\title{
A Comparison of Neutral Gas Models for Divertor Plasmas
}

\author{
M. E. Rensink, L. Lodestro, G. D. Porter, T. D. Rognlien \\ Lawrence Livermore National Laboratory, Livermore, CA 94550, USA \\ and D. P. Coster \\ Max-Planck-Institut für Plasmaphysik, Garching, FRG
}

\section{Introduction}

Fluid models of both plasma and neutral components are often used for simulating divertor plasmas. In parameter regimes where the collisional mean free path becomes comparable to divertor dimensions the fluid approximation may not be valid. In this report we compare results from fluid and Monte Carlo models of the neutral component coupled to the fluid plasma model in the UEDGE code [1]. Our objective is to benchmark the fluid neutrals model against a Monte Carlo model, the EIRENE code [2], for a range of plasma parameters and geometric configurations.

In section 2 we briefly describe the fluid models for plasma and neutrals in UEDGE. The general features of the results for attached and detached plasmas are compared in section 3. In section 4 we examine the spatial distributions of the neutral particle densities and plasma source terms in more detail.

\section{Model Description}

\subsection{Fluid Plasma Model}

The UEDGE code is a fully implicit 2-D edge plasma transport code. It solves the classical Braginskii transport equations [3] for plasma density, parallel momentum and thermal ion and electron energy transport along the magnetic field, $\mathbf{B}$, and assumes anomalous diffusive transport across $\mathbf{B}$. An arbitrary number of multi-charge-state impurity ion species may be modeled, but for the problems described in this report we assume a pure deuterium plasma. At the core boundary we fix the plasma density and power flow into the plasma. At the divertor target we impose a sonic flow boundary condition [4] on the parallel component of the plasma velocity and specify fixed sheath transmission factors [5] for electron and ion energy fluxes. At the radial mesh boundaries (except in the core region) we use either zero-radial-derivative or zero-radial-flux boundary conditions.

\subsection{Fluid Neutrals Model}

A full Navier Stokes model for neutral transport has been applied to dense recombining divertor plasmas by Knoll [6]. The UEDGE code uses a reduced Navier-Stokes model [7] with a single momentum equation for the neutrals. Molecules coming off the divertor target and sidewalls are assumed to instantaneously break up into neutral atoms, so only a single neutral species is present for a pure hydrogen plasma. Although molecules are not explicitly represented in the fluid model, the electron energy loss associated with their breakup is included in the electron energy balance equation. The neutral fluid equations are written in poloidal $(\mathrm{x})$ and radial $(\mathrm{y})$ coordinates, suppressing the 
metric factors. The continuity equation for the neutral atoms is

$$
\frac{\partial n_{n}}{\partial t}+\frac{\partial \Gamma_{n x}}{\partial x}+\frac{\partial \Gamma_{n y}}{\partial y}=n_{n} n_{e} K^{i}-n_{i} n_{e} K^{r}
$$

The neutral atom parallel momentum equation is

$$
\begin{gathered}
\frac{\partial}{\partial t}\left(m n_{n} v_{n \|}\right)+\frac{\partial}{\partial x}\left(m v_{n \|} \Gamma_{n x}-\eta_{n x} \frac{\partial v_{n \|}}{\partial x}\right)+\frac{\partial}{\partial y}\left(m v_{n \|} \Gamma_{n y}-\eta_{n y} \frac{\partial v_{n \|}}{\partial y}\right) \\
=-\frac{B_{x}}{B} \frac{\partial P_{n}}{\partial x}-m n_{n} n_{i} K^{c x}\left(v_{n \|}-v_{i \|}\right)+m n_{e}\left(n_{i} K^{r} v_{i \|}-n_{n} K^{i} v_{n \|}\right)
\end{gathered}
$$

where $B_{x} / B$ is the ratio of the poloidal to total magnetic field and $K^{i}, K^{r}$ and $K^{c x}$ are rate coefficients $\langle\sigma \cdot v\rangle$ for ionization, recombination and charge exchange. The neutral, ion and electron densities are $n_{n}, n_{i}$ and $n_{e}$; the ions and neutrals are assumed to share a common temperature, $T$. the poloidal and radial particle fluxes are

$$
\begin{gathered}
\Gamma_{n x}=\frac{B_{x}}{B} n_{n} v_{n \|}-D_{n}\left(\frac{\partial n_{n}}{\partial x}+\frac{n_{n}}{T} \frac{\partial T}{\partial x}\right) \\
\Gamma_{n y}=-D_{n}\left(\frac{\partial n_{n}}{\partial y}+\frac{n_{n}}{T} \frac{\partial T}{\partial y}\right)
\end{gathered}
$$

Flux limits can be imposed on the transport of particles, parallel momentum and thermal energy. The neutral particle diffusion and viscosity coefficients are

$$
\begin{aligned}
& D_{n}=\frac{\sqrt{T / m}}{n_{i} K^{c x}+n_{e} K^{i}} \\
& \eta_{n}=\frac{n_{n} \sqrt{m T}}{n_{i} \sigma_{c x}+n_{n} \sigma_{n n}}
\end{aligned}
$$

At the divertor target the recycling boundary conditions (for an orthogonal plate) are

$$
\begin{aligned}
\Gamma_{n x} & =-R_{p} \Gamma_{i x} \\
v_{n \|} & =-R_{m} c_{s} \\
Q_{n x} & =-R_{E} Q_{i x}
\end{aligned}
$$

where $c_{s}$ is the plasma sound speed, $Q_{i x}$ is the total ion energy flux, and $R_{p}, R_{m}$ and $R_{E}$ are particle, momentum and energy recycling parameters. At sidewalls with albedo $A_{w}$ the boundary conditions are

$$
\Gamma_{n y}= \pm\left(1-A_{w}\right) n_{n} \sqrt{\frac{T}{2 \pi m}}
$$

and zero-shear for $v_{n \|}$. 


\subsection{Coupling Algorithms}

With the fluid neutrals model that is built into the UEDGE code we use fully implicit numerical methods to efficiently obtain self-consistent steady-state solutions for the strongly coupled plasma+neutral system. With the EIRENE Monte Carlo neutrals code [8] we use a numerically explicit time-dependent coupling procedure in which plasma source terms for particles, momentum and energy due to interaction with neutrals are derived from the Monte Carlo results. Similar coupled fluid plasma and Monte Carlo neutrals models, such as B2-EIRENE [9,10], have been routinely used for simulating divertor plasmas in several existing and proposed fusion experiments.

\section{Self-Consistent (Plasma+Neutral) Steady States}

We compare the model results for a simple Cartesian box geometry, illustrated in Figure 1, that simulates the outer half of the edge plasma in a single-null divertor configuration. The walls coincide with the flux surfaces at the radial edges of the mesh. The particle recycling coefficient at the plate is 0.98 and the wall albedos are 0.99 . For the simulations described in this report the core plasma density is $7 \times 10^{19} \mathrm{~m}^{-3}$, the anomalous diffusion coefficients for density and energy are $D=0.5 \mathrm{~m}^{2} / \mathrm{s}$ and $\chi_{e, i}=0.7 \mathrm{~m}^{2} / \mathrm{s}$, respectively. The electron and ion sheath transmission factors are 4.0 and 2.5 , respectively. Power input across the innermost core flux surface boundary varied from $8 \mathrm{~kW}$ to $94 \mathrm{~kW}$.

We simulate both attached and detached plasmas by varying the input power across the core boundary. For each value of the input power we run the coupled plasma-neutrals system to a steady state. The transition from attached to detached plasmas is shown in Figures 2-4 for both the fluid and Monte Carlo neutrals models. At high input power, the plasma density and temperature are high enough to immediately ionize neutrals coming off the plate. At lower input power, the peak plasma temperature at the target plate drops (Figure 2) and the neutrals can penetrate further upstream before being ionized. The plasma "detaches" from the plate and the peak of the ionization source is located upstream some finite distance from the target plate. This usually happens when the plasma plate temperature is in the range 1-2 eV. In this temperature regime, volume recombination may reduce the plasma density and ion current (Figure 3) to the divertor target. The poloidal distance from the target plate to the ionization front increases strongly with a reduction in input power for both neutrals models. This can be seen in Figure 4 as an increase in the particle throughput for decreasing input power; as the ionization front moves upstream away from the target plate it exposes a larger area of the sidewalls to the high neutral particle pressure in the detached region and results in more particles being pumped by the sidewalls. At very low power and/or with reduced pumping, the ionization front may be displaced all the way up to the $\mathrm{x}$-point and a localized marfe-like structure may develop or a steady state may not even exist. With the Monte Carlo neutrals model the plasma seems to detach at a somewhat higher power, but the fluid model contains some free parameters which could be adjusted to reduce this discrepancy. While the Monte Carlo model uses TRIM code data to characterize the plasma-surface interaction the fluid model uses the adjustable particle, parallel momentum and energy recycling coefficients at the divertor target plate. [The flux limit 
coefficients may also be regarded as adjustable parameters.] For the results shown here the EIRENE wall and target plate material was molybdenum, but user-supplied multiplicative factors for the particle recycling coefficients ensure the same particle removal rate as in the fluid model. The fluid model results can be sensitive to the momentum and energy recycling parameters, particularly in the detached plasma regime. This is shown in Figures 2-4 where $x$ 's indicate the range of results for $-0.2<R_{m}<+0.2$. Non-zero values of these parameters represent some degree of backscattering [11] for energetic ions incident on the target plate. The solid curves in Figures 2-4 corresponds to no backscattering, i.e., all ions are recycled as thermal molecules.

\section{Spatial Distribution of the Neutrals}

Some insight regarding the differences in the two models can be obtained by examining the spatial distribution of the neutral particle densities and plasma source terms. We run both neutral models to steady state in the same fixed plasma background. The models were compared for both attached and detached plasma regimes. In Figures 5-6 we plot the neutral particle densities and net plasma particle source from plate recycling and volume recombination along a flux surface just outside the separatrix for a detached plasma. The net particle sources are very similar, but the atomic neutral density just in front of the targer plate is much lower (by more than a factor of 10 in our example). In the Monte Carlo model, most neutrals are launched from the divertor plate as molecules rather than atoms and subsequently break up into relatively energetic ( $4 \mathrm{eV}$ ) FranckCondon atoms at a distance of about one molecular mean-free path from the plate; in the fluid model, all neutrals are launched as atoms with the local ion temperature (typically less than $1 \mathrm{eV}$ for detached plasmas). The lower atomic neutral density with the Monte Carlo model means that ions flowing toward the plate will experience less frictional drag (due to both charge exchange and elastic scattering on the neutrals) so the momentum transfer between plasma and neutrals will be reduced.

\section{Summary and Conclusions}

Fluid and Monte Carlo neutrals models both produce the same signs of plasma detachment: thermal collapse of the electron temperature at the divertor plate, a reduction in the ion current to the plate due to volume recombination, and a tendency for the ionization front of a detached plasma to move upstream toward the $\mathrm{x}$-point. With the Monte Carlo neutral model the plasma seems to detach at higher input power and the particle throughput is higher. For detached plasmas, with pumping, there is a stable position for the ionization front between the target plate and the $\mathrm{x}$-point. With the fluid model the position of the ionization front is sensitive to the plasma momentum recycling coefficient. For detached plasmas, the molecular and atomic neutral densities near the target plate are lower in the Monte Carlo model than in the fluid model; this could alter the momentum coupling with the plasma and affect the position of the ionization front.

\section{Acknowledgments}

Work performed under the auspices of the United States Department of Energy by the Lawrence Livermore National Laboratory under contract number W-7405-ENG-48. 


\section{References}

[1] Rognlien, T.D., et al., Contr. Plasma Phys. 34 (1994) 362.

[2] Reiter, D., J. Nucl. Mat. 196-198 (1992) 80.

[3] Braginskii, S.I., Transport processes in a plasma, Reviews of Plasma Physics, Vol. I, Ed. M.A. Leontovich (Consultants Bureau, New York, 1965), p. 205.

[4] Chodura, R., Proceedings of NATO Advanced Study Institute on Physics of PlasmaWall Interactions in Controlled Fusion, p 99, Val-Morin, Quebec, Canada (1984).

[5] Stangeby, P., Proceedings of NATO Advanced Study Institute on Physics of PlasmaWall Interactions in Controlled Fusion, p 41, Val-Morin, Quebec, Canada (1984).

[6] Knoll, D., Phys. Plasmas 3 (1996) 293.

[7] Wising, F., et al., Contr. Plasma Phys. 36 (1996) 136.

[8] Reiter, D., et.al., J. Nucl. Mat. 220-222 (1995) 987.

[9] Schneider, R., et.al., J. Nucl. Mat. 196-198 (1992) 810.

[10] Coster, D.P., et.al., Contr. Plasma Phys. 36 (1996) 150.

[11] Behrisch, R., et.al., Proceedings of NATO Advanced Study Institute on Physics of Plasma-Wall Interactions in Controlled Fusion, p 413, Val-Morin, Quebec, Canada (1984)!

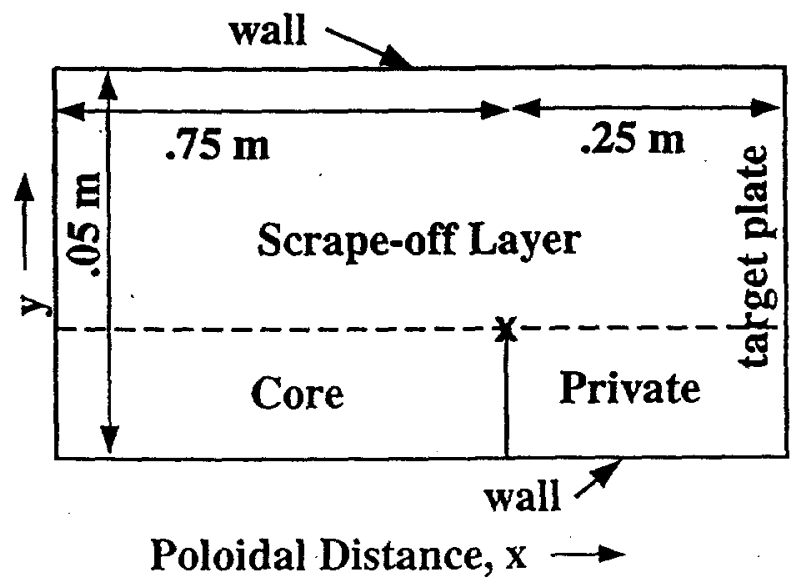

Figure I
Plate Temperature (eV)

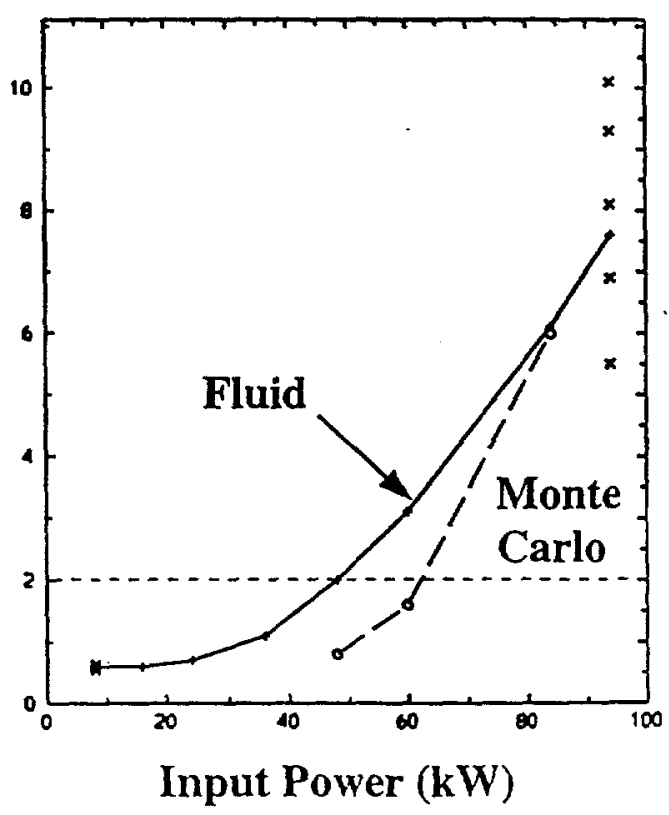

Figure 2 


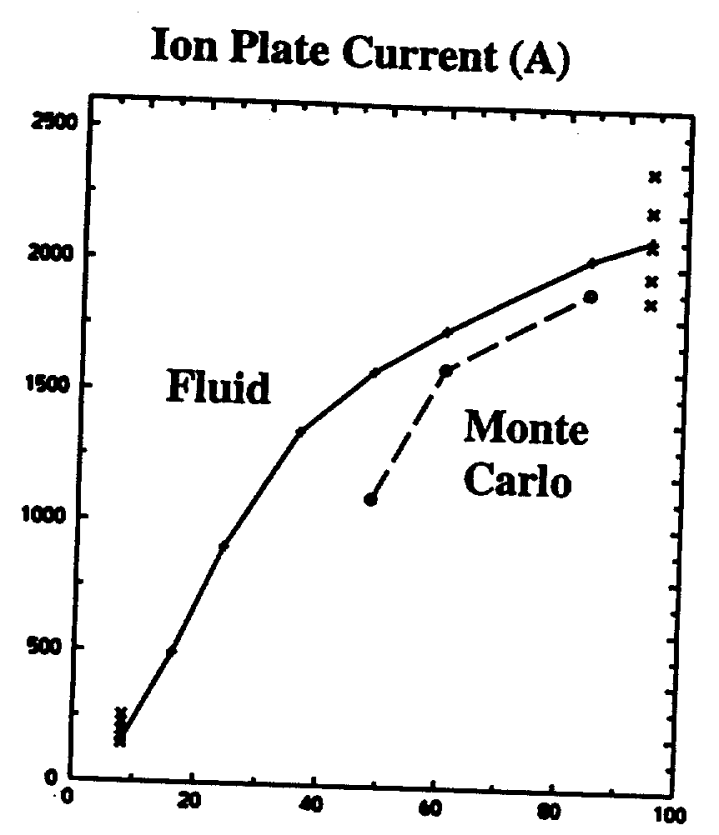

Input Power (kW)

Figure 3

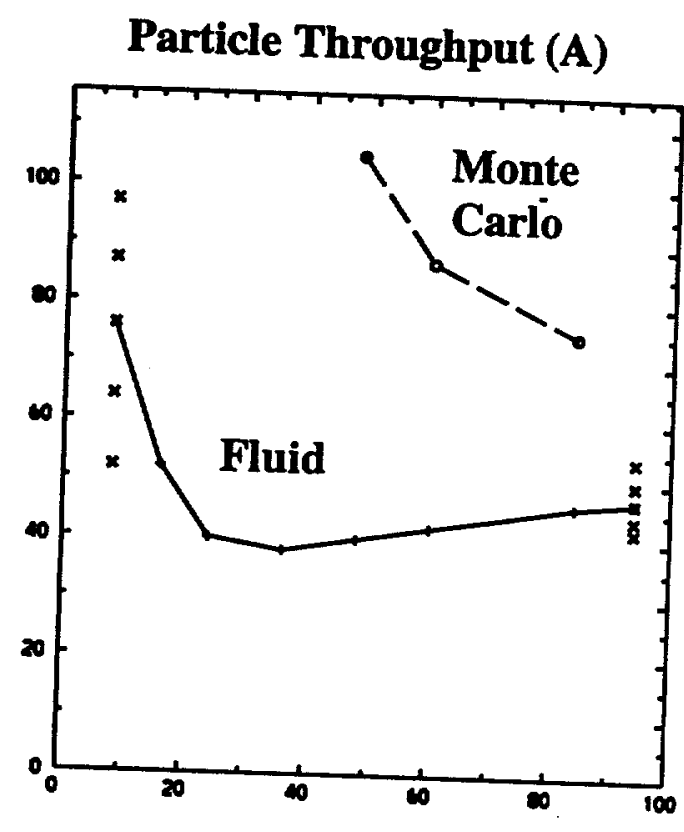

Input Power (kW)

Figure 4

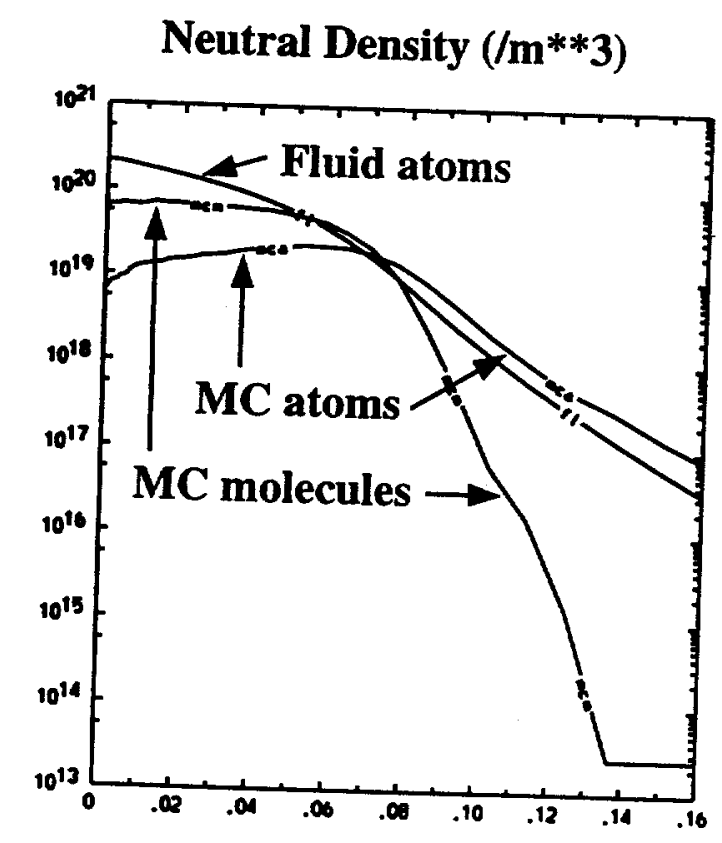

Distance (m) from Plate

Figure 5

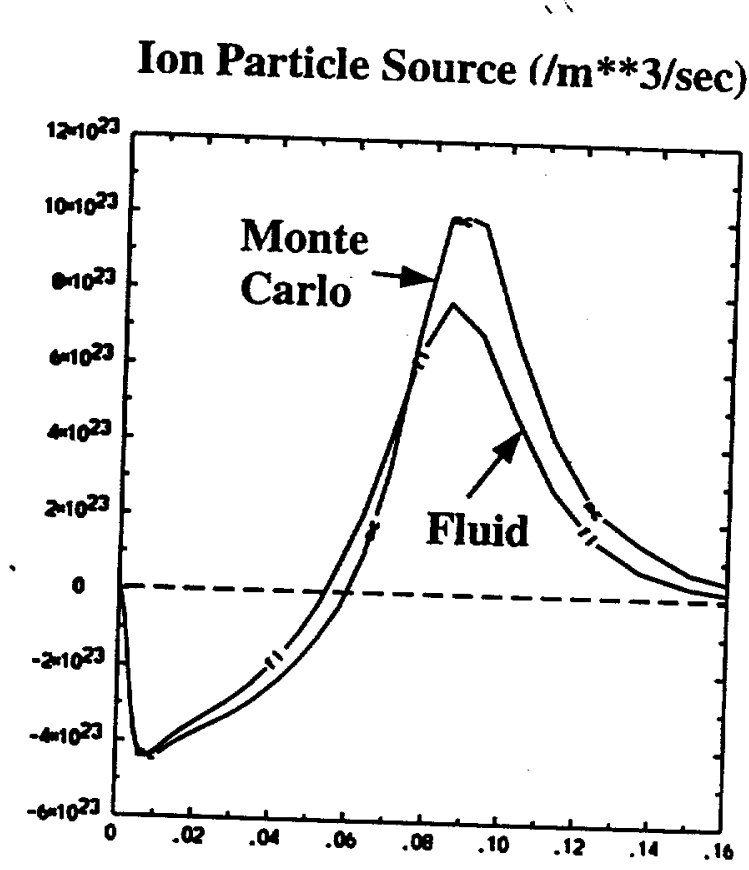

Distance (m) from Plate

Figure 6 


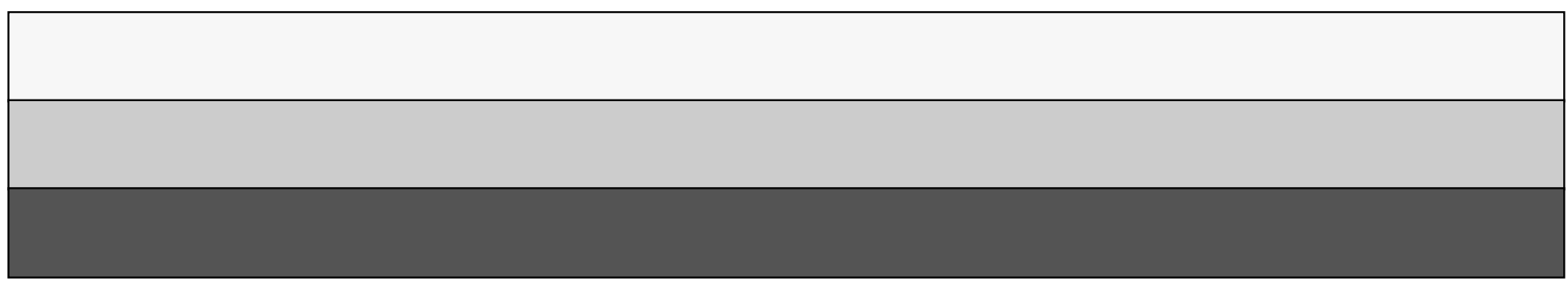

